Article

\title{
Overexpression of the Jojoba Aquaporin Gene, ScPIP1, Enhances Drought and Salt Tolerance in Transgenic Arabidopsis
}

\author{
Xing Wang ${ }^{1}$, Fei Gao ${ }^{2}$, Jie Bing ${ }^{1}$, Weimin Sun ${ }^{1}$, Xiuxiu Feng ${ }^{1}$, Xiaofeng Ma ${ }^{1}$, Yijun Zhou ${ }^{2, *}$ \\ and Genfa Zhang $1, *$ (D) \\ 1 Beijing Key Laboratory of Gene Resource and Molecular Development, College of Life Sciences, Beijing \\ Normal University, Beijing 100875, China; xingwang163@163.com (X.W.); bingjie@bnu.edu.cn (J.B.); \\ 201021200018@bnu.edu.cn (W.S.); 15249220644@163.com (X.F.); mxf510332934@163.com (X.M.) \\ 2 College of Life and Environmental Sciences, Minzu University of China, Beijing 100081, China; \\ gaofei@muc.edu.cn \\ * Correspondence: zhouyijun@muc.edu.cn (Y.Z.); gfzh@bnu.edu.cn (G.Z.); \\ Tel.: +86-10-6893-2922 (Y.Z.); +86-10-5880-9453 (G.Z.)
}

Received: 27 November 2018; Accepted: 26 December 2018; Published: 3 January 2019

\begin{abstract}
Plasma membrane intrinsic proteins (PIPs) are a subfamily of aquaporin proteins located on plasma membranes where they facilitate the transport of water and small uncharged solutes. PIPs play an important role throughout plant development, and in response to abiotic stresses. Jojoba (Simmondsia chinensis (Link) Schneider), as a typical desert plant, tolerates drought, salinity and nutrient-poor soils. In this study, a PIP1 gene (ScPIP1) was cloned from jojoba and overexpressed in Arabidopsis thaliana. The expression of ScPIP1 at the transcriptional level was induced by polyethylene glycol (PEG) treatment. ScPIP1 overexpressed Arabidopsis plants exhibited higher germination rates, longer roots and higher survival rates compared to the wild-type plants under drought and salt stresses. The results of malonaldehyde (MDA), ion leakage (IL) and proline content measurements indicated that the improved drought and salt tolerance conferred by ScPIP1 was correlated with decreased membrane damage and improved osmotic adjustment. We assume that $S c P I P 1$ may be applied to genetic engineering to improve plant tolerance based on the resistance effect in transgenic Arabidopsis overexpressing ScPIP1.
\end{abstract}

Keywords: Simmondsia chinensis; aquaporin; PIP1; drought stress; salt stress

\section{Introduction}

Aquaporins transport water, $\mathrm{CO}_{2}$ and small neutral solutes through the plasma and intracellular membranes of cells [1-3]. They play a central role in regulating water transport in many physiological and developmental processes, including cell elongation, stomatal regulation, seed germination, reproductive growth and stress responses in plants [4,5]. Based on conserved amino acid sequences and intron positions, AQPs are divided into five groups: plasma membrane intrinsic proteins (PIPs), tonoplast intrinsic proteins (TIPs), nodulin-like plasma membrane intrinsic proteins (NIPs), small intrinsic proteins (SIPs) and X intrinsic proteins (XIPs) [6]. The plasma membrane intrinsic proteins present in the plasma membrane regulate water movement between cells and coordinate with TIPs to maintain cell water balance.

Drought, high salinity, low temperature and other abiotic stresses could result in water loss in plants, which seriously limits plant growth, development and productivity worldwide. Water movement is a key physiological process of plants and must be tightly regulated under drought and salt stress conditions [7-9]. The transport of water is controlled by both symplastic and apoplastic 
pathways [10]. The symplastic pathway in which aquaporins (AQPs) play a central role is efficient for transporting water across membranes [11-14].

The expression regulation, biological activity and localization of PIPS are regulated by abiotic stresses, plant hormones and light [15-20]. Arabidopsis PIP transcripts are generally downregulated upon gradual drought stress in leaves, with the exception of AtPIP1;4 and AtPIP2;5, which are upregulated [21]. AtPIP2;6 and constitutively expressed and not significantly affected by the drought stress [21]. Under drought stress, the accumulation of NtPIP1;1 and NtPIP2;1 transcripts was significantly decreased, but only that of the $N t A Q P 1$ transcript was increased [22]. The level of VfPIP1 mRNA had obviously increased from 0.5 to $2.0 \mathrm{~h}$ after 20\% PEG6000PEG treatment [23]. During $100 \mathrm{mmol} / \mathrm{L} \mathrm{NaCl}$ treatment, GmPIP1;6 reduced expression initially, then the expression was increased in root and leaves after 3 days [24]. OsPIP1;3 was induced by 2.5 -fold at $2 \mathrm{~h}$ of $250 \mathrm{mmol} / \mathrm{L} \mathrm{NaCl}$ treatment and then gradually declined; whereas the expression of OsPIP1;1 increased by about two-fold constantly [25].

By means of transgenic approaches, some studies have demonstrated that overexpressing a PIP1 gene in plants confers tolerance to abiotic stresses based on the roles of PIP1s in response to the adverse environment [23-28]. The overexpression of TaAQP7 in tobacco enhanced drought tolerance [27], and the overexpression of $T a A Q P 8$, another wheat PIP gene, induces salt stress tolerance in tobacco when compared with wild-type plants [28]. However, not all PIPs confer tolerance to abiotic stresses in transgenic plants. The overexpression of HvPIP2;1 resulted in the transgenic rice being more sensitive to drought and salt stresses because of an increased transpiration rate and decreased intrinsic water-use efficiency [29]. Previous studies have demonstrated that the different effects of overexpressing PIPs depended on the sources and isoforms of these genes. Therefore, specific PIPS, which confer plants better growth and development status under abiotic stresses, are potential genetic resources in agronomy and crop science.

However, there is no research on the overexpression of the PIP1 gene from desert plants, which have many precious agronomic traits. Jojoba, as a typical desert plant, has very rare agronomic traits, such as tolerance to high-temperature, drought, salt and poor soil environments. Exploring germplasm resources, cloning stress response genes and researching gene functions will have important value. SCPIP1 is a drought-induced gene, suggesting its role in response to water deficit, and there are no reports on this gene so far. In this study, we first cloned the full-length ScPIP1 gene encoding a plasma membrane intrinsic protein in Jojoba. Based on a transgenic approach, ScPIP1 overexpressing Arabidopsis plants were generated, and their resistance was evaluated at physiological and biochemical levels. Additionally, the cause of improved tolerance was reported in our study.

\section{Results}

\subsection{ScPIP1 Gene Encodes a PIP1 Subgroup of AQPs in Jojoba}

Our laboratory has screened 385 unique ESTs based on a water stress induced suppression subtractive hybridization (SSH) cDNA library of jojoba. We found that the EST sequence (Genbank accession number: DV752738) of aquaporin was highly repeated, indicating that this gene was regulated by water deficit [30]. By using the rapid amplification of cDNA ends (RACE) method, a full-length cDNA of the aquaporin-like gene was isolated from cDNA prepared from jojoba leaves and designated as ScPIP1. Analysis of the sequence showed that ScPIP1 cDNA is $1244 \mathrm{bp}$ in length and contains a $5^{\prime}$-noncoding region (74 bp) and an open reading frame ( $855 \mathrm{bp}$ ) that encodes 284 amino acids and a 3'-noncoding region ( $315 \mathrm{bp}$ ). The analysis of the amino acid sequence revealed that ScPIP1 had $93 \%$ sequence identity with mipB of Mesembryanthemum crystallinum. ScPIP1 contains six putative transmembrane $\alpha$-helices, two 'NPA' motifs and an MIP signal sequence (SGxHxNPAVT) in loop B (Figure 1). Phylogenetic analysis of ScPIP1 with mipB [M. crystallinum] (AAA93521), AtPIP1;4 [A. thaliana] (NP_567178), KsPIP1 [Knorringia sibirica] (ACC85598), DcPIP1 [Dianthus caryophyllus] (BAI94500), VvPIP1;1 [Vitis vinifera] (AEZ35024), GhAQP1 [Gossypium hirsutum] (ABD63904), pm28b [Spinacia oleracea] (CAB56217), CsPIP1-4 [Camelina sativa] (AEH76328) and 
PAQ1 [Raphanus sativus] (BAA32777) showed that ScPIP1 and mipB belong to the same class. These results suggest that the ScPIP1 gene isolated in this study is a member of the PIP1 subfamily in Jojoba.

A SCPIP1[STMMONDSIA_CHINENSIS (LINK) SCHNEIDER] MIPB [MESEMBRYANTHEMUM_CRYSTALLINUM] [AAA93521]

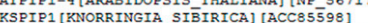
DCPIP1 [DIANTHUS CARYOPHYLLUS] [BAI 94500 VVPIP1; 1 [VITIS_VINIFERA] [AE235024] PNos (SPTWCTA O CSPIP1-4 [CAMEIINA SAIIVA] [AEH7 632 PAQ1 [RAPHANUS_SATIVUS] [BAA32777]

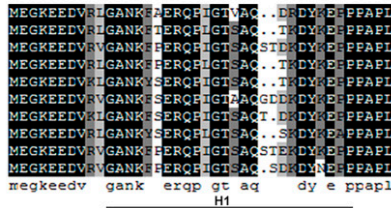

SCPIP1 [STMMONDSIA CHINENSIS (LINK) SCHNE IDER]
MIPB [MESEMBRYANTHEMUM CRYSTALINUM] [AAA93521]

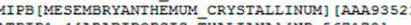

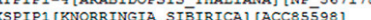
DCPIP1 [DIANTHUS CÄRYOPHYLLUS] [BAI94500] VVPIP1; 1 [VITIS_VINIFERA] [AE235024]

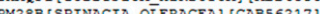
CSPIP1-4 [CAMEIINA SATIVA] [AEH76328] PAQ1 [RAPHANUS_SATIVUS] [BAA32777] Consensus

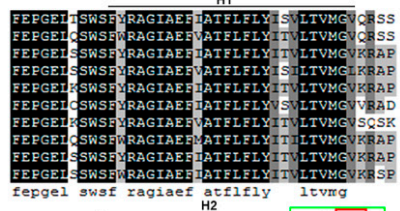

$$
\begin{aligned}
& 78 \\
& 78 \\
& 80 \\
& 78 \\
& 78 \\
& 80 \\
& 79 \\
& 78 \\
& 80 \\
& 79
\end{aligned}
$$

SCPIP1 [STMMONDSIA CHINENSIS (LINR) SCHNEIDER] MIPD MELAN WANTHEMUM CRYSTALLINUM] [AAA9352 SSPIP1 [RNORRINGIA SIBIRICA] [ACC85598] DCPIP1 (DIANTHUS_CÄRYOPHYLIUS] [BAI 94500 ] GPIP1; 1 [VITIS VINIFERA] [AE 235024]
GHAQR1 [GOSSYPIUM HIRSUTUM] [ABD 63904] 88 [SPTNACTA OİERACEA] [CAB56217] CSPIP1-4 [CAMEIINNA_SATIVA] [AEH7 6328] Consensus

SCPIP1 [STMMONDSIA_CHINENSIS (LINR) SCHNEIDER] MIPB [MESEMBRYANTHEMUM_CRYSTALLINUM] [AAA93521] ATP IP1-4 [ARADIDOFSIS _ MALIAANA] [NP 567170 ] DCPIP1 [DTANTHUS CÄRYOPHYLIUS] [BAI94500] VVPIP1; 1 [VITIS_VINIFERA] [AE235024] GHAQP1 [GOSSYPIUM HIRSUTUM] [ABD63904] CSPIP1-4 CAMET TIMA SATIVA] [AEH7632 PAQ1 [RAPHANUS SATIVUS] [BAA32777] Consensus

SCPIP1 [SIMMONDSIA CHINENSIS (LINK) SCHNEIDER] MIPB [MESEMBRYANTHEMUM CRYSTALL INUM] [AAA9352 ATPIP1-4 [ARABIDOFSIS THALIANA] [NP 567178 ]
KSPI P1 [KNORRINGIA SIBIRICA] [ACC 55598 ] DCPIP1 [DIANTHUS_CÄRYOPHYLLUS] [BAI94500] GHAPI; 1 [VITIS VINIFERA] [AE Z35024]
GHAQ1 [GOSSYPIUMA HIRSTUM] [ABD 63904] PM28B [SPINACIA_OIERACEA] [CAB56217] PAQ1 [RAPHANUS SATIVUS] [BAA32777] Consensus

SCPIP1 [STMMONDSIA_CHINENSIS (IINK) SCHNEIDER] MI PB [MESEMBRYANTHEMUM, CRYSTAIL INUM] [AAA93521] ATPIP1 4 [ARABIDOPSIS_THALIANA] [NP 567178 DCPIP1 [DIANTHUS CĀRYOPHYLLUS] [BAI94500) VVPIP1; 1 [VITIS VINIFERA] [AEZ35024] GAQRE [GOSSYP TUMA HIRSUTUM] [ABD63904] LTNA SATIVA] [AFH76328] PAQ1 [RAPHANUS SATIVUS] [BAA32777] Consensus

SCPIP1 [STMMONDSIA CHINENSIS (LINK) SCHNEIDER] MIPB [MESEMBRYANTHEMUMA CRYSTALLINUM] [AAA93521]
ATPI P1 - 4 [ARABIDOPSIS_THALIANA] [NP_567178] DCPIP1 [DIANTHUS CĀRYOPHYIIUS] [BAI94500) VVPIP1; 1 [VITIS VINIFERA] [AE235024] GHAQP1 [GOSSYPIUM_HIRSUTUM] [ABD63904] PM28B [SPINACIA OLERACEA] [CAB56217] PAO1 [RAPHANUS_SATIVUS] [BAA32777] Consensus

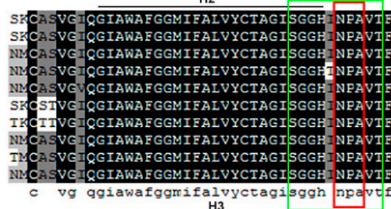

SCPIP1 [SIMMONDSIA_CHINENSIS (LINK) SCHNEIDER] MIPB [MESEMBRYANTHEMUM_CRYSTALIINUM] [AAA93521] ATPIP1-4 [ARABIDOPSIS_THALIANA] [NP_567178] KSPIP1 [RNORRINGIA_SIBIRICA] [ACC85598] VVPIP1; [ [VITIS VINT FERA] [AE 235024] GHAQP1 [GOSSYPTUMM_HIRSUTUM] [ABD63904] PM28B[SPINACIA_OLERACEA] [CABS6217] CSPIP1-4 [CAMELINA SAT IVA] [AEH7 632
PAQ1 [RAPHANUS SATIVUS] [BAA32777] Consensus

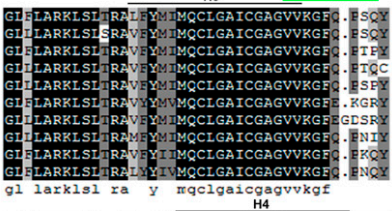

$$
\begin{aligned}
& 118 \\
& 118 \\
& 120 \\
& 118 \\
& 118 \\
& 120 \\
& 119 \\
& 118 \\
& 120
\end{aligned}
$$
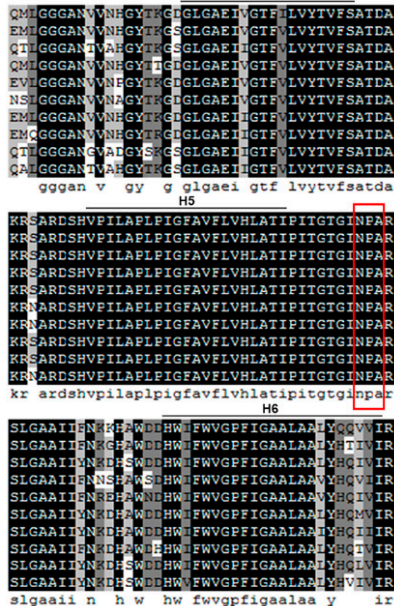

$$
\begin{aligned}
& 157 \\
& 157 \\
& 159 \\
& 157 \\
& 157 \\
& 159 \\
& 159 \\
& 157 \\
& 159 \\
& 158
\end{aligned}
$$

。

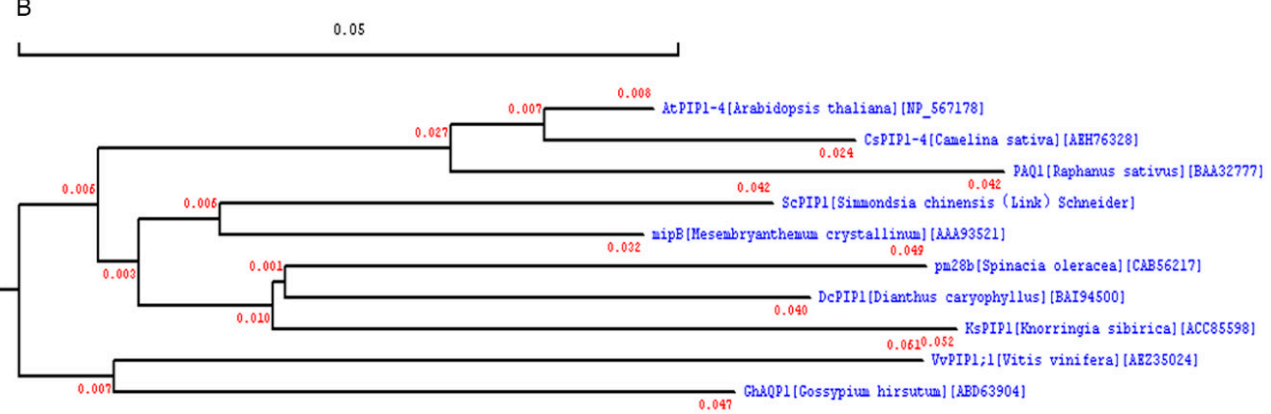

Figure 1. Alignment and phylogenetic analysis of ScPIP1 with other known PIP proteins. Amino acid sequences were aligned by DNAMAN software (A). The conserved amino acid residues in all proteins 
are highlighted in grey. Two conserved NPA motifs (red frame) and the MIP signal sequence (green frame) are boxed. The six putative transmembrane $\alpha$-helices (H1-H6) are shown by lines. Phylogenetic analysis of ScPIP1 was conducted by the maximum likelihood method (B). Values at nodes in the phylogenetic tree in Figure 1B represent bootstrap values indicating branching probability per 1000 replicates.

\subsection{ScPIP1 Is Upregulated in Jojoba Leaves after PEG Treatment}

To investigate the expression mode of ScPIP1 in jojoba leaves under PEG stress, six-week old jojoba seedlings were treated with 30\% PEG6000 for 0, 3, 6, 12, 24 or $48 \mathrm{~h}$ and total RNA was extracted from jojoba leaves. Moreover, mRNA was converted to cDNA before being subjected to real-time quantitative polymerase chain reaction (qRT-PCR) analysis. ScPIP1 transcripts were steadily upregulated within $6 \mathrm{~h}$ and downregulated at $12 \mathrm{~h}$. The highest expression levels appeared at $24 \mathrm{~h}$, however, the expression of ScPIP1 transcripts reduced at $48 \mathrm{~h}$ (Figure 2).

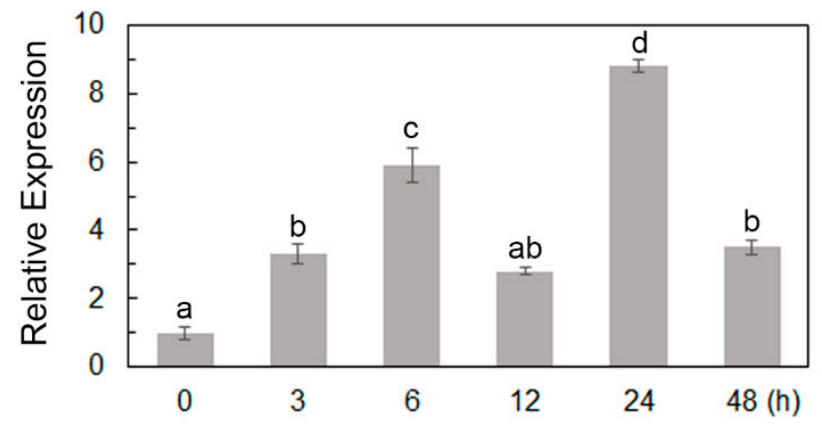

Figure 2. Regulation of ScPIP1 expression under PEG stress. Characterization of the qRT-PCR analysis of ScPIP1 from jojoba plants treated with 30\% PEG6000 for 0, 3, 6, 12, 24 or $48 \mathrm{~h}$. The data are shown as the means \pm standard deviation (SD) of 3 biological replicates. Significant differences were determined by one-way ANOVA followed by Duncan's multiple range test at $p<0.05$.

\subsection{Phenotypic Analysis of Transgenic Arabidopsis Lines Overexpressing ScPIP1}

To determine the role of ScPIP1 in plants, ScPIP1 was transformed into the pCAMBIA1302 vector under the control of the $35 \mathrm{~S}$ promoter. Through the floral-dip transformation of Arabidopsis, three homozygous $\mathrm{T}_{3}$ transgenic lines (L7, L8 and L11) were selected on MS medium containing $50 \mathrm{mg} / \mathrm{L}$ hygromycin. ScPIP1 overexpressing transgenic lines L7, L8 and L11 exhibited longer root lengths and better growth status than the wild type (WT) (Figure 3A-E). These results suggest that the overexpression of ScPIP1 influences root elongation and leaf development under optimum growth conditions.

\subsection{Overexpression of ScPIP1 in Arabidopsis Enhances Tolerance to Drought Stress}

To investigate the drought tolerance of transgenic Arabidopsis overexpressing ScPIP1, four-week-old WT and transgenic Arabidopsis were treated without watering for 20 days and then rewatered. The transgenic lines exhibited higher survival rates than the WT (Figure 4A,C). The transgenic Arabidopsis had a lower water loss rate (Figure 4B). To determine the role of ScPIP1 in transgenic Arabidopsis under drought stress, WT and transgenic seeds and seedlings were treated with mannitol. The transgenic lines showed higher germination rates and longer roots than the WT under mannitol treatment (Figure 4D-G). These results revealed that the transgenic lines exhibited better growth, higher germination rates, longer roots, higher survival rates and lower water loss rates compared to the WT under drought and osmotic stresses, indicating that the overexpression of ScPIP1 improves the tolerance of Arabidopsis to drought and osmotic stresses. 
A

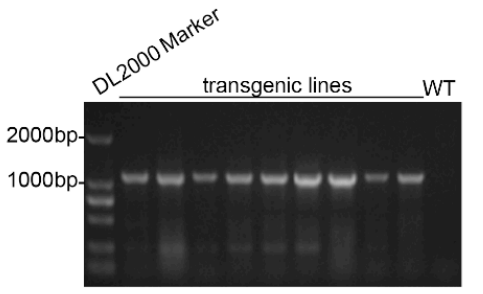

C

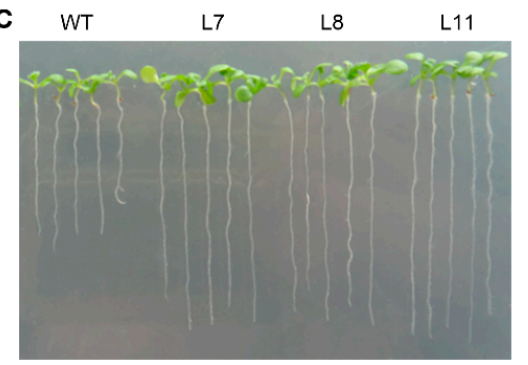

E

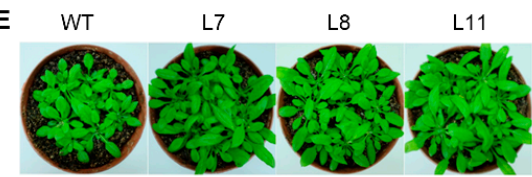

B

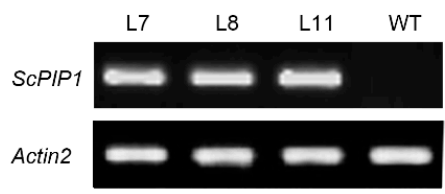

D

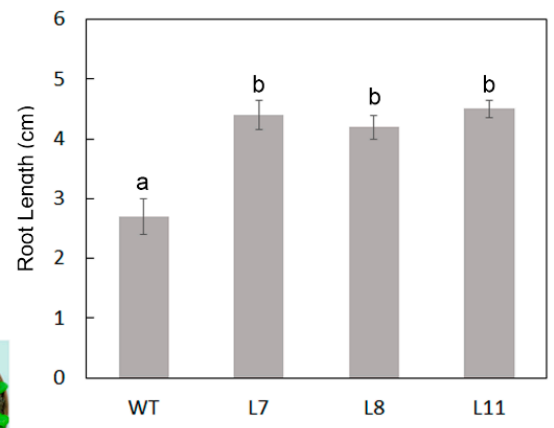

Figure 3. Molecular and phenotype analysis of ScPIP1 overexpressing Arabidopsis lines. Genomic DNA PCR analysis (A) of WT and transgenic lines. Semiquantitative RT-PCR characterization (B) of ScPIP1 and AtActin2 in WT and transgenic lines. Images (C) and the results of statistical analyses (D) of the root lengths of nineteen-day-old WT and transgenic lines. (E) Images of four-week-old transgenic lines and WT under optimum growth conditions. The data are presented as the means \pm SD of 3 biological replicates. Significant differences were determined by one-way ANOVA followed by Duncan's multiple range test at $p<0.05$.

\subsection{Overexpression of ScPIP1 in Arabidopsis Decreases MDA Content and IL, and Increases Proline Accumulation under Drought Stress}

Increased drought and osmotic tolerance in the transgenic Arabidopsis compared to the WT allowed us to investigate the physiological and biochemical differences between WT and ScPIP1 overexpressing transgenic lines. Malonaldehyde (MDA), ion leakage (IL) and proline content were quantified in the WT and ScPIP1 overexpressing transgenic lines under optimum and drought conditions. The results revealed that there was no significant difference in MDA, IL and proline content between the WT and transgenic lines under optimum growth condition. However, compared to the WT, reduced MDA and IL, and higher proline content were observed in the transgenic lines under drought treatment (Figure 5A-C). These results indicate that the overexpression of ScPIP1 reduces lipid peroxidation and maintains the accumulation of osmotic substances under drought stress.

\subsection{Overexpression of ScPIP1 Enhances Tolerance to Salt Stress}

To examine the role of $S c P I P 1$ during salt stress, WT and ScPIP1 overexpressing transgenic lines were treated with $\mathrm{NaCl}$. When four-week-old WT and ScPIP1 overexpressing transgenic lines were treated with $300 \mathrm{mM} \mathrm{NaCl}$ for $20 \mathrm{~d}$, ScPIP1 overexpressing transgenic lines showed a higher survival rate and more green leaves compared to the WT (Figure 6A,B). To understand the role of ScPIP1 in seed germination and root development, the WT and ScPIP1 overexpressing transgenic lines were subjected to $50 \mathrm{mM}$ to $150 \mathrm{mM} \mathrm{NaCl}$ treatment. The transgenic seeds exhibited higher germination rates and the transgenic seedlings showed longer root lengths than those of WT (Figure 6C-F). These results indicate that $S C P I P 1$ overexpressing transgenic lines showed better growth, higher survival rates, higher germination rates and longer roots than those of the WT plants. Therefore, ScPIP1 overexpressing transgenic lines were more tolerant to salt stress than the WT. 


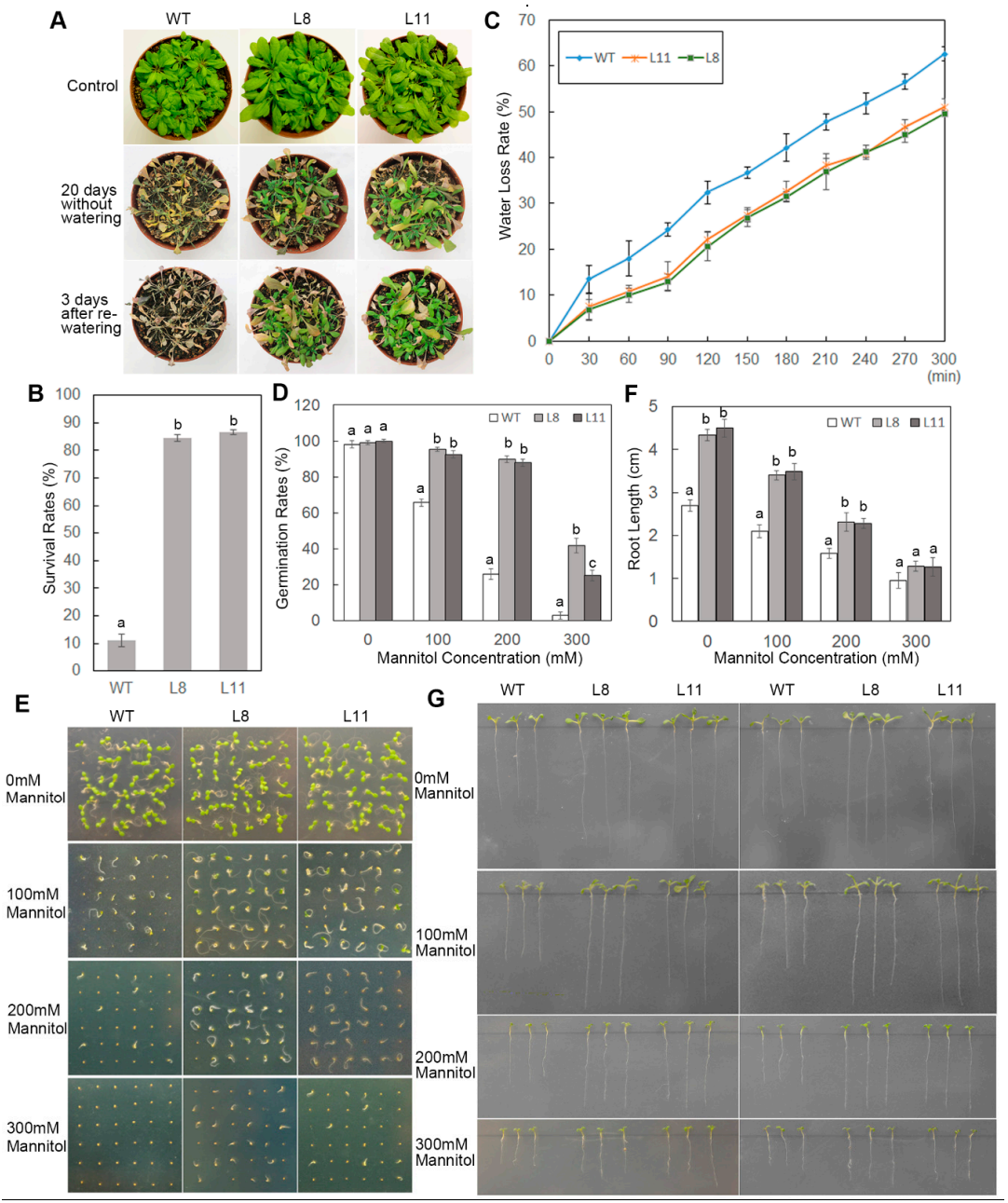

Figure 4. Phenotypes of ScPIP1 overexpressing transgenic lines under drought. Images (A) and survival rates (B) of the four-week-old WT and transgenic lines under drought stress for 20 days. (C) Water loss rates of the leaves of WT and transgenic lines in vitro. Results of the statistical analyses (D) and images (E) of the germination test of the WT and transgenic lines under mannitol treatments for 5 days. Results of the statistical analyses (F) and images (G) of the root lengths of the WT and transgenic lines under mannitol treatments for 15 days. The data are presented as the means \pm SD of 3 biological replicates. Significant differences were determined by one-way ANOVA followed by Duncan's multiple range test at $p<0.05$.
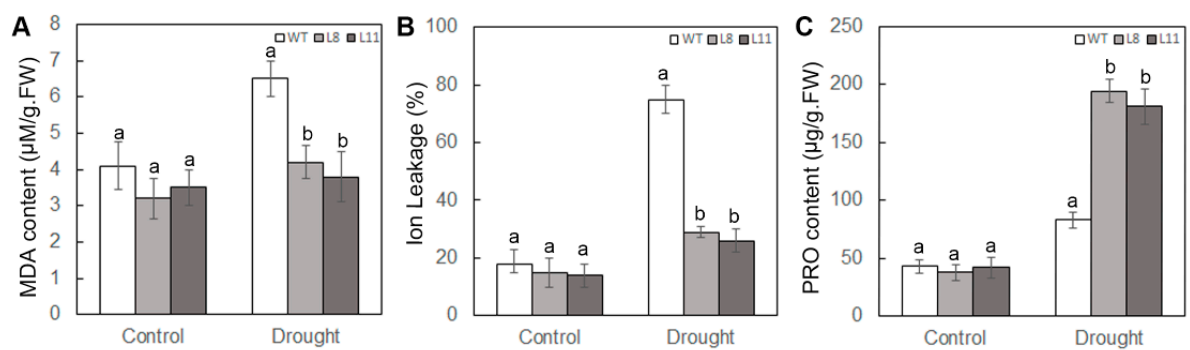

Figure 5. Analysis of the MDA, IL and proline contents of the WT and ScPIP1 overexpressing transgenic lines under drought stress. The malonaldehyde content (A), ion leakage (B) and proline content (C) measured in the leaves of WT and transgenic lines that were well watered or without watered for 20 days. The data are presented as the means \pm SD of 3 biological replicates. Significant differences were determined by one-way ANOVA followed by Duncan's multiple range test at $p<0.05$. 


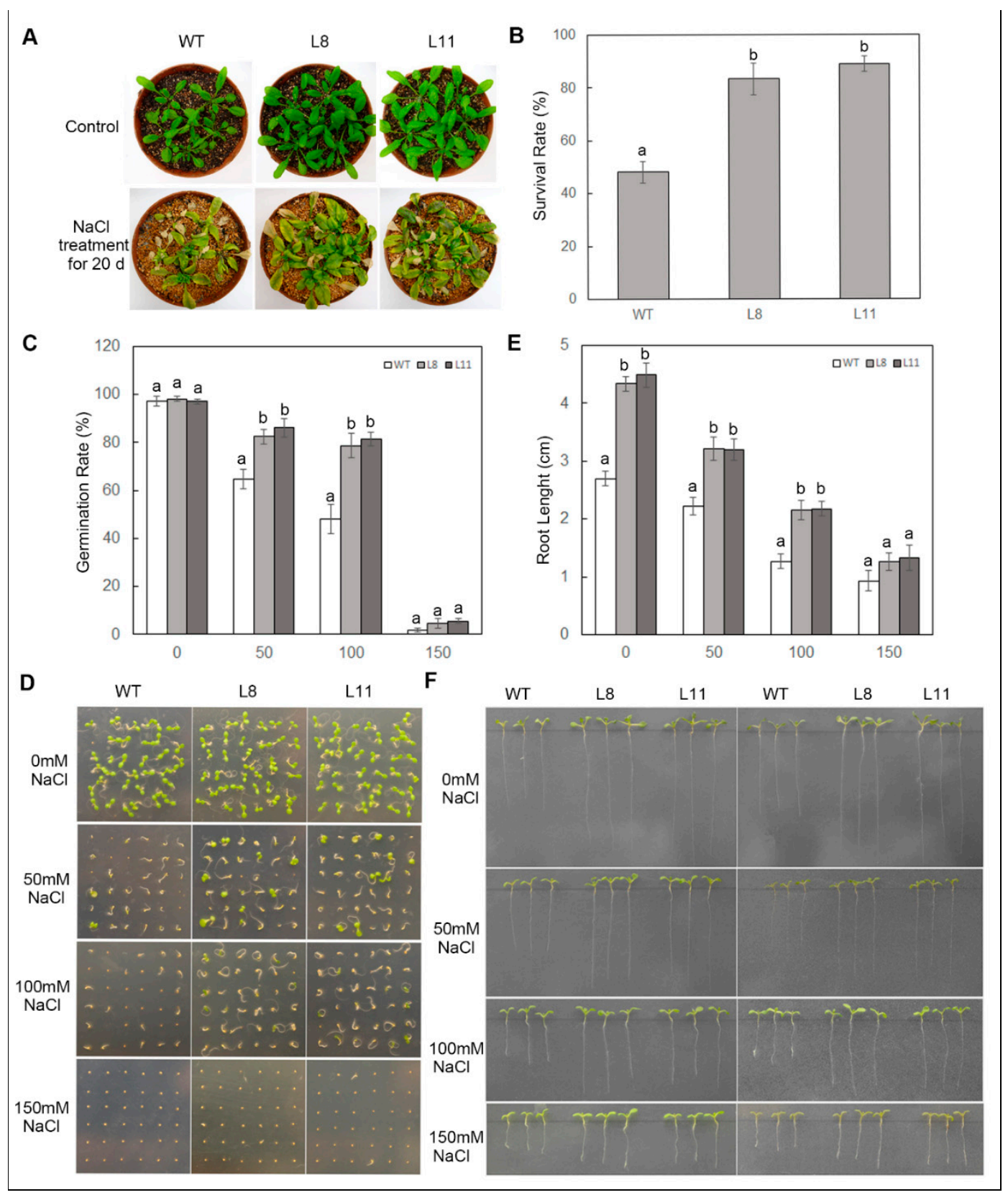

Figure 6. Phenotypes of ScPIP1 overexpressing transgenic lines under salt stress. Images (A) and survival rates (B) of the four-week-old WT and transgenic lines under salt stress for 20 days. Results of the statistical analyses (C) and images (D) of the germination test of the WT and transgenic lines under $\mathrm{NaCl}$ treatments for 5 days. Results of the statistical analyses (E) and images (F) of the root lengths of the $\mathrm{WT}$ and transgenic lines under $\mathrm{NaCl}$ treatment for 15 days. The data are presented as the means \pm SD of 3 biological replicates. Significant differences were determined by one-way ANOVA followed by Duncan's multiple range test at $p<0.05$.

\subsection{Overexpression of ScPIP1 in Arabidopsis Decreases MDA Content and IL, and Increases Proline} Accumulation under Salt Stress

To study the role of ScPIP1 in improving the salt tolerance of transgenic Arabidopsis compared to that in WT plants, the MDA, IL and proline contents were quantified in the WT and ScPIP1 overexpressing transgenic lines under optimum and salt conditions. Similar to the drought stress experiments, there was no significant difference in the MDA, IL and proline contents between the WT and the transgenic lines under optimum growth conditions. However, under $\mathrm{NaCl}$ treatment, 
the transgenic lines exhibited reduced MDA and IL, and higher proline content compared to the WT (Figure 7A-C), suggesting less membrane damage caused by lipid peroxidation and more accumulation of osmotic substances in the transgenic lines.
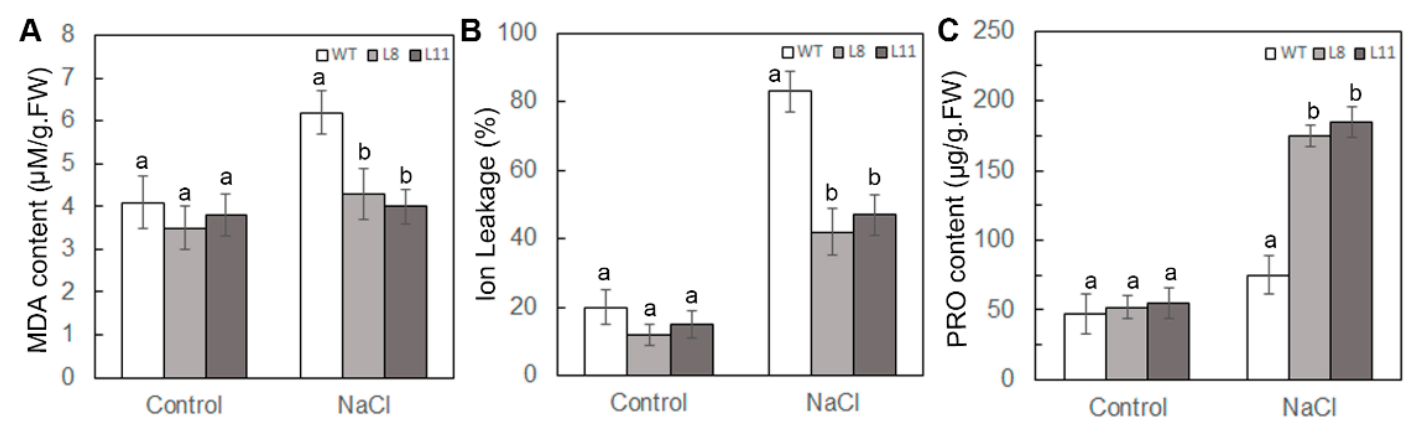

Figure 7. Analysis of the MDA, IL and proline contents of the WT and ScPIP1 overexpressing transgenic lines under salt stress. Malonaldehyde content (A), ion leakage (B) and proline content (C) measured in the leaves of WT and transgenic lines under optimum and salt conditions. The data are presented as the means \pm SD of 3 biological replicates. Significant differences were determined by one-way ANOVA followed by Duncan's multiple range test at $p<0.05$.

\section{Discussion}

\subsection{Overexpression of ScPIP1 Enhances Plant Resistance to Drought and Salt Stresses}

It has been reported that PIPs are involved in abiotic stress resistance [3,5,25-31]. In the present study, we found that the expression level of ScPIP1 in jojoba leaves was upregulated under PEG treatment, indicating that SCPIP1 plays a positive role in response to osmotic and drought stresses. To further examine the function of ScPIP1 under drought and salt stress, we generated three homozygous ScPIP1 overexpressing transgenic Arabidopsis lines. The transgenic seeds, seedlings and adult plants were more tolerant to drought and salt stresses compared to the WT plants. Consistent with previous studies $[15,16,21-25]$, these results demonstrated that overexpression of some specific PIP genes enhances abiotic stress resistance.

\subsection{Response of ScPIP1 to Abiotic Stress Involved in Reducing Membrane Damage}

Drought and salt stresses cause the rapid accumulation of reactive oxygen species (ROS), resulting in membrane damage and oxidation [32,33]. MDA, a product of lipid peroxidation caused by ROS, can be used to evaluate ROS induced damage in plants [34]. Ion leakage is also a significant indicator of membrane damage. Therefore, MDA content and IL were measured to examine the role of ScPIP1 in decreasing the membrane damage caused by lipid peroxidation under optimum, drought or salt conditions. The overexpression of ScPIP1 led to reduced MDA content and IL compared with that in the WT, suggesting that ScPIP1 overexpressing transgenic plants may experience less membrane damage and lipid peroxidation under drought or salt conditions. Previous studies have reported that compared to the control, the overexpression of TaAQP7 in transgenic tobacco (Nicotiana tabacum L.) exhibited lower levels of MDA and IL under drought stress [27] and the overexpression of TaAQP8 in transgenic tobacco showed reduced MDA and IL under salt stress [28]. MaPIP1;1 overexpressing transgenic Arabidopsis plants exhibited reduced MDA and IL under drought and salt stresses [35]. OsPIP2;7 overexpressing transgenic rice (Oryza sativa L.) plants showed decreased IL under chilling stress [36]. In conclusion, these studies show that PIP acts as an important gene subgroup in reducing the MDA content and IL, accordingly decreasing membrane damage under various abiotic stresses. PIPs can facilitate water transportation through the membrane rapidly under optimum or drought and salt conditions, thereby maintaining a relatively healthy physiological status, which may contribute to reducing protein and lipid peroxidation, and decreasing membrane damage. 


\subsection{Response of ScPIP1 to Abiotic Stress Involved in Improving Osmotic Adjustment}

The ability to maintain water balance is important for plants to resist drought and salt stresses, and PIPs play vital roles in the plant water balance process under water deficit conditions. We found that compared to the WT plants, ScPIP1 overexpressing transgenic plants showed better growth and a higher survival rate under drought and salt conditions, suggesting a positive response of SCPIP1 to water deficit. When plants experience drought and salt stresses, the accumulation of osmotic substances is considered a way to sustain osmotic adjustment. Proline, a compatible osmolyte, contributes to maintaining plant cell osmotic balance and enhancing cellular protection when plants suffered from stresses [37]. The overexpression of SCPIP1 in transgenic plants increased the accumulation of proline compared to the WT plants under drought and salt stresses, indicating that ScPIP1 may contribute to maintaining osmotic adjustment under water deficit conditions. We considered that the improved osmotic adjustment may also be related to the reduced membrane damage conferred by SCPIP1 under drought and salt stresses.

\section{Materials and Methods}

\subsection{Plant Materials, Growth Conditions and Abiotic Stress Treatments}

Jojoba seedlings were obtained from the jojoba stem apex culture tube (SY03). Jojoba rooted seedlings were transferred to soil watered with Hoagland's solution in growth chambers $\left(28{ }^{\circ} \mathrm{C}\right.$; $110 \mu \mathrm{mol} \cdot \mathrm{m}^{-2} \cdot \mathrm{s}^{-1}$ light intensity; 16 -h light $/ 8$-h dark cycle; $50 \%$ relative humidity). For the expression levels assay, six-week-old jojoba rooted seedlings in soil were watered with Hoagland's solution supplemented with $30 \%$ PEG6000 for 0, 3, 6, 12, 24 or $48 \mathrm{~h}$.

Arabidopsis thaliana ecotype Columbia (Col-0) was used as the WT control in the study and all transgenic lines were generated in the background of Col-0. Seeds were sterilized with $0.1 \%(w / v)$ $\mathrm{HgCl}_{2}$ for $10 \mathrm{~min}$, washed 5 times with sterile water, sown on Murashige and Skoog (MS) medium (3\% $(w / v)$ sucrose, $0.7 \%(w / v)$ agar) and vernalized at $4{ }^{\circ} \mathrm{C}$ for 3 days in the dark. Ten-day-old seedlings were transferred to pots filled with a mixture of soil and sand $(3: 1)$ and grown in a chamber $\left(22^{\circ} \mathrm{C}\right.$; $110 \mu \mathrm{mol} \cdot \mathrm{m}^{-2} \cdot \mathrm{s}^{-1}$ light intensity; $16-\mathrm{h}$ light $/ 8$-h dark cycle; $70 \%$ relative humidity). For germination test, the surface sterilized seeds were directly sown on MS medium supplemented with 0, 100, 200, $300 \mathrm{mM}$ mannitol or supplemented with $0,50,100,150 \mathrm{mM} \mathrm{NaCl}$ for 5 days. Radicle protrusion was used as a criterion for seed germination. For the root length assay, four-day-old seedlings were transferred to MS medium supplemented with 0, 100, 200, $300 \mathrm{mM}$ mannitol or 0, 50, 100, $150 \mathrm{mM}$ $\mathrm{NaCl}$ for 15 days. For the stress tolerance study, four-week-old plants in pots were unwatered or watered with $300 \mathrm{mM} \mathrm{NaCl}$ for 20 days. Then, photos were taken, and germination rates, root length and survival rates were calculated.

\subsection{Cloning and Bioinformatics Analysis of Jojoba ScPIP1}

An EST sequence (Accession Number: DV752738) of aquaporin was identified from drought induced suppression subtractive hybridization (SSH) cDNA library of eight-year-old female jojoba (SY03) [30]. By rapid-amplification of cDNA ends (RACE), the full-length cDNA of aquaporin was cloned from cDNA of jojoba leaves. For $5^{\prime} \mathrm{RACE}$, the forward primer sequence was $5^{\prime}$-tcagggggtcacattaaccc- $3^{\prime}$ and the reverse primer sequence was $5^{\prime}$-acgagcacttcgcttagcatcag- $3^{\prime}$. For $3^{\prime}$ RACE, the forward primer sequence was $5^{\prime}$-gaagatgattgcagcaccga- $3^{\prime}$, and the reverse primer sequence was $5^{\prime}$-gcttccagccatctcagtatcag- $3^{\prime}$. The EST sequences and the sequences of the $5^{\prime}$ RACE and $3^{\prime}$ RACE products were spliced together. Based on the sequences of the $5^{\prime}$ and $3^{\prime}$ ends, a pair of identical primers was designed ( $5^{\prime}$-atgggcaccatctccattgtca- $3^{\prime}$ and $5^{\prime}$-cggaaacgaaacatcatacagtcgg- $\left.3^{\prime}\right)$ to amplify the ScPIP1 full-length sequence. The bioinformatics analysis of ScPIP1 was evaluated by DNAMAN software and BLAST (http:/ /blast.ncbi.nlm.nih.gov/Blast.cgi). 


\section{3. $q R T-P C R$}

Total RNA was extracted from jojoba leaves under various time treatments with 30\% PEG6000 using the RNeasy Plant Mini Kit (Qiagen, Amsterdam, The Netherlands). Total RNA ( $1 \mu \mathrm{g})$ from each sample was converted into cDNA by reverse transcription using the RNA PCR Kit (TaKaRa) according to the manufacturer's instructions. qRT-PCR was conducted on an ABI 7500 system (Applied Biosystems, New York, NY, USA) using TransStart ${ }^{\mathrm{TM}}$ Green qRT-PCR SuperMix Kit (TransGen, Beijing, China). Sc16S rRNA (GenBank Accession: AJ505848) was used as a reference gene to normalize the relative transcriptional abundance and to minimize different copy numbers of cDNA templates. The control sample was conferred a value of 1 . All the data were calculated and analyzed from three independent samples based on the $2^{-\Delta \Delta \mathrm{Ct}}$ method [38]. The primers for Sc16S $r R N A$ (5'-acaaggtagccgtactggaa- $3^{\prime}$ and $5^{\prime}$-gccgagaaacgaaagaagac- $\left.3^{\prime}\right)$ and ScPIP1 (5'-cttccagccatctcagtatcag- $3^{\prime}$ and $5^{\prime}$-agcacttcgcttagcatcag- $\left.3^{\prime}\right)$ had high specificity according to the results.

\subsection{Plant Transformation and Generation of Transgenic Plants}

The ScPIP1 ORF with PstI and AvrII restriction sites was amplified by specific primers ( $5^{\prime}$-aactgcagatggagggcaaggaggaggatg- $3^{\prime}$ and $5^{\prime}$-cgcctaggcttagacttgaaggggatagcc- $\left.3^{\prime}\right)$. The PCR products were cloned into the modified pCAMBIA1302-GFP expression vector to generate the 35S::ScPIP1-GFP fusion vector. The fusion vector was transferred into the Agrobacterium tumefaciens Smith \& Townsend strain GV3101 and cultivated at a suitable concentration. Using the floral dip method, transgenic plants were generated [39]. Then, the seeds were selected by $40 \mathrm{mg} / \mathrm{L}$ hygromycin B on MS medium and the seedlings were identified by PCR amplification with specific primers ( $5^{\prime}$-tcacttcttggtccttctaccg- $3^{\prime}$ and $5^{\prime}$-ttcaccttgatgccgttct-3'). Three T3 homozygous lines were generated and examined by PCR and semi-quantitative RT-PCR. AtActin2 (GenBank Accession: NM_112764) was used as an internal control.

\subsection{Rate of Water Loss}

Forty integrated leaves were extracted from the four-week-old WT plants and transgenic lines, and then weighed immediately (fresh weight, FW). All samples were placed on respective open Petri dishes in a growth chamber $\left(22{ }^{\circ} \mathrm{C}\right.$, humidity $\left.45 \%\right)$ and were weighed every half hour (desiccated weights, DW) for $6 \mathrm{~h}$. The water loss rates were calculated according to the formula: water loss rate $(\%)=(\mathrm{FW}-\mathrm{DW}) / \mathrm{FW} \times 100[40]$.

\subsection{MDA, IL and Proline Content Measurements}

Four-week-old plants were not watered for 20 days or watered with $350 \mathrm{mM} \mathrm{NaCl}$ treatment for $15 \mathrm{~d}$, then the leaves were collected to measure the MDA, IL and proline contents. The MDA content was measured using a malondialdehyde assay kit (Nanjing Jiancheng Bioengineering Institute, China). IL was determined based on the method described by Jiang and Zhang [41]. The samples were cut into strips and cultivated in $10 \mathrm{~mL}$ distilled water at room temperature for $8 \mathrm{~h}$, and the initial conductivity (C1) was measured by a conductivity meter (DDBJ-350, Shanghai, China). Then the samples were boiled for $10 \mathrm{~min}$. The conductivity (C2) was measured when the samples were cooled to room temperature. IL was calculated based on the equation: IL $(\%)=C 1 / C 2 \times 100$. The proline content was measured using a proline assay kit (Nanjing Jiancheng Bioengineering Institute, China).

\subsection{Statistical Analysis}

The data are presented as means \pm SD and were compared using the software Statistical Product and Service Solutions (SPSS) with one-way ANOVA followed by Duncan's multiple range test at the significant level of $p<0.05$. 


\section{Conclusions}

The findings of this study demonstrate the gene function of ScPIP1 in response to drought and salt stresses. The overexpression of ScPIP1 led to improved tolerance to drought and salt stresses by reducing membrane damage and improving osmotic adjustment (Figure 8). These results further illustrate the physiological mechanisms of transgenic plant resisting abiotic stresses and demonstrate the role of PIPS in decreasing membrane damage and maintaining osmotic balance. Furthermore, we must declare that the results from the experiments on transgenic Arabidopsis may not be the same when overexpressing SCPIP1 in other species. Therefore, more studies on the function and role of ScPIP1 in jojoba or other species are required.

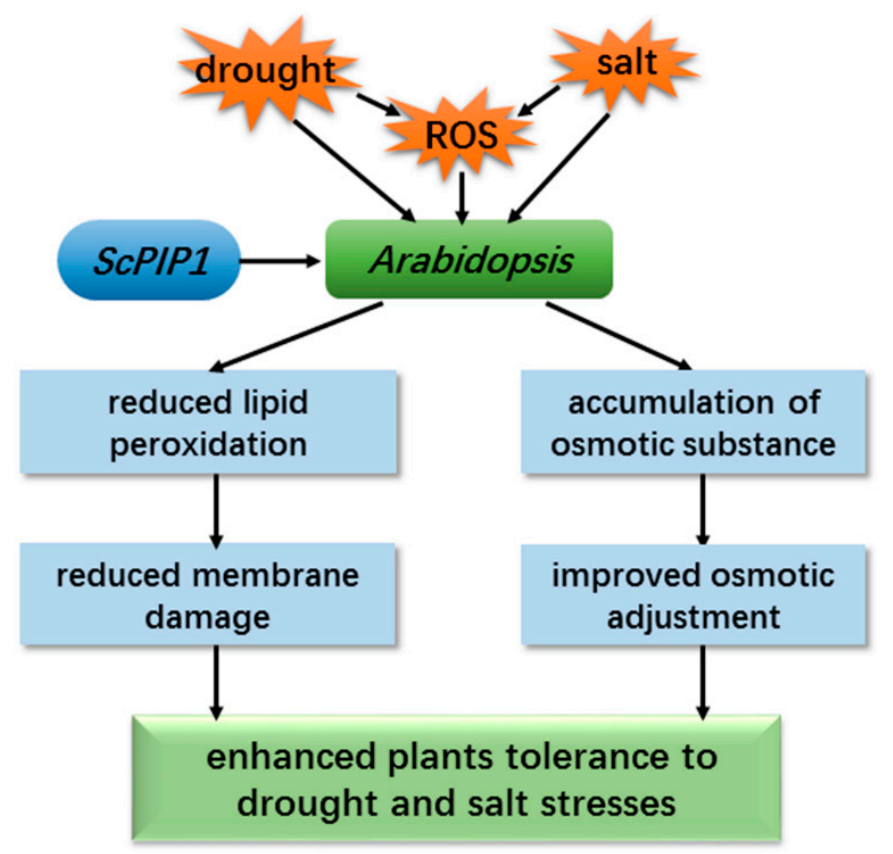

Figure 8. Effects of ScPIP1 heterologous expression on tolerance to drought and salt stresses of Arabidopsis. Physiological responses to drought and salt stresses enhance the plant tolerance.

Author Contributions: Conceptualization, X.W., F.G., G.Z. and Y.Z.; Data curation, X.W., J.B., W.S. and X.F.; Formal analysis, X.W., W.S. and X.F.; Funding acquisition, G.Z.; Methodology, X.W., J.B., W.S. and X.F.; Project administration, F.G.; Resources, J.B.; Software, X.W., J.B., W.S., X.F. and X.M.; Supervision, F.G., G.Z. and Y.Z.; Writing-original draft, X.W.; Writing-review \& editing, X.W., F.G., G.Z. and Y.Z.

Funding: This work was supported by the National Natural Science Foundation of China $(31270365,31470399$ and 31872672) and the Open fund from Beijing Key Laboratory of Gene Resource and Molecular Development.

Conflicts of Interest: The authors declare no conflict of interest.

\section{References}

1. Uehlein, N.; Lovisolo, C.; Siefritz, F.; Kaldenhoff, R. The tobacco aquaporin NtAQP1 is a membrane $\mathrm{CO}_{2}$ pore with physiological functions. Nature 2003, 425, 734-737. [CrossRef]

2. Kaldenhoff, R.; Fischer, M. Aquaporins in plants. Acta Physiol. 2006, 187, 169-176. [CrossRef]

3. Sade, N.; Gebretsadik, M.; Seligmann, R.; Schwartz, A.; Wallach, R.; Moshelion, M. The role of tobacco Aquaporin1 in improving water use efficiency, hydraulic conductivity, and yield production under salt stress. Plant Physiol. 2010, 152, 245-254. [CrossRef]

4. Eisenbarth, D.A.; Weig, A.R. Dynamics of aquaporins and water relations during hypocotyl elongation in Ricinus communis L. seedlings. J. Exp. Bot. 2005, 56, 1831-1842. [CrossRef] 
5. Gao, Z.; He, X.; Zhao, B.; Zhou, C.; Liang, Y.; Ge, R.; Shen, Y.; Huang, Z. Overexpressing a putative aquaporin gene from wheat, TaNIP, enhances salt tolerance in transgenic Arabidopsis. Plant Cell Physiol. 2010, 51, 767-775. [CrossRef]

6. Maurel, C.; Verdoucq, L.; Luu, D.T.; Santoni, V. Plant aquaporins: Membrane channels with multiple integrated functions. Annu. Rev. Plant Biol. 2008, 59, 595-624. [CrossRef]

7. Bray, E.A. Molecular responses to water deficit. Plant Physiol. 1993, 103, 1035-1040. [CrossRef]

8. Cheeseman, J.M. Mechanisms of salinity tolerance in plants. Plant Physiol. 1988, 8, 547-550. [CrossRef]

9. Blumwald, E. Sodium transport and salt tolerance in plants. Curr. Opin. Cell Biol. 2000, 12, 431-434. [CrossRef]

10. Aharon, R.; Shahak, Y.; Wininger, S.; Bendov, R.; Kapulnik, Y.; Galili, G. Overexpression of a plasma membrane aquaporin in transgenic tobacco improves plant vigor under favorable growth conditions but not under drought or salt stress. Plant Cell 2003, 15, 439-447. [CrossRef]

11. Suga, S.; Komatsu, S.; Maeshima, M. Aquaporin isoforms responsive to salt and water stresses and phytohormones in radish seedlings. Plant Cell Physiol. 2002, 43, 1229-1237. [CrossRef]

12. Lian, H.L.; Yu, X.; Ye, Q.; Ding, X.; Kitagawa, Y.; Kwak, S.S.; Su, W.A.; Tang, Z.C. The role of aquaporin RWC3 in drought avoidance in rice. Plant Cell Physiol. 2004, 45, 481-489. [CrossRef]

13. Vera-Estrella, R.; Barkla, B.J.; Bohnert, H.J.; Pantoja, O. Novel regulation of aquaporins during osmotic stress. Plant Physiol. 2004, 135, 2318-2329. [CrossRef]

14. Amodeo, G.; Dorr, R.; Vallejo, A.; Stuka, M.; Parisi, M. Radial and axial water transport in the sugar beet storage root. J. Exp. Bot. 1999, 50, 509-516. [CrossRef]

15. Ji, Y.J.; Dong, G.K.; Kim, Y.O.; Jin, S.K.; Kang, H. An expression analysis of a gene family encoding plasma membrane aquaporins in response to abiotic stresses in Arabidopsis thaliana. Plant Mol. Biol. 2004, 54, 713-725.

16. Fricke, W.; Akhiyarova, G.; Wei, W.; Alexandersson, E.; Miller, A.; Kjellbom, P.O.; Richardson, A.; Wojciechowski, T.; Schreiber, L.; Veselov, D.; et al. The short-term growth response to salt of the developing barley leaf. J. Exp. Bot. 2006, 57, 1079-1095. [CrossRef]

17. North, G.B.; Nobel, P.S. Heterogeneity in water availability alters cellular development and hydraulic conductivity along roots of a desert succulent. Ann. Bot. 2000, 85, 247-255. [CrossRef]

18. Horie, T.; Kaneko, T.; Sugimoto, G.; Sasano, S.; Panda, S.K.; Shibasaka, M.; Katsuhara, M. Mechanisms of water transport mediated by PIP aquaporins and their regulation via phosphorylation events under salinity stress in barley roots. Plant Cell Physiol. 2011, 52, 663-675. [CrossRef]

19. Chaumont, F.; Moshelion, M.; Daniels, M.J. Regulation of plant aquaporin activity. Biol. Cell 2005, 97, 749-764. [CrossRef]

20. Bienert, G.P.; Schjoerring, J.K.; Jahn, T.P. Membrane transport of hydrogen peroxide. Biochim. Biophys. Acta 2006, 1758, 994-1003. [CrossRef]

21. Alexandersson, E.; Fraysse, L.; Sjövall-Larsen, S.; Gustavsson, S.; Fellert, M.; Karlsson, M.; Johanson, U.; Kjellbom, P. Whole gene family expression and drought stress regulation of aquaporins. Plant Mol. Biol. 2005, 59, 469-484. [CrossRef]

22. Mahdieh, M.; Mostajeran, A.; Horie, T.; Katsuhara, M. Drought stress alters water relations and expression of PIP-type aquaporin genes in Nicotiana tabacum plants. Plant Cell Physiol. 2008, 49, 801-813. [CrossRef]

23. Cui, X.H.; Hao, F.S.; Chen, H.; Chen, J.; Wang, X.C. Expression of the Vicia faba VfPIP1 gene in Arabidopsis thaliana plants improves their drought resistance. J. Plant Res. 2008, 121, 207-214. [CrossRef]

24. Zhou, L.; Wang, C.; Liu, R.; Han, Q.; Vandeleur, R.K.; Du, J.; Tyerman, S.; Shou, H.X. Constitutive overexpression of soybean plasma membrane intrinsic protein GmPIP1;6 confers salt tolerance. BMC Plant Biol. 2014, 14, 181. [CrossRef]

25. Guo, L.; Wang, Z.Y.; Lin, H.; Cui, W.E.; Chen, J.; Liu, M.; Chen, Z.L.; Qu, L.J.; Gu, H. Expression and functional analysis of the rice plasma-membrane intrinsic protein gene family. Cell Res. 2006, 16, 277-286. [CrossRef]

26. Peng, Y.H.; Arora, R.; Li, GW.; Wang, X.; Fessehaie, A. Rhododendron catawbiense plasma membrane intrinsic proteins are aquaporins, and their overexpression compromises constitutive freezing tolerance and cold acclimation ability of transgenic Arabidopsis plants. Plant Cell Environ. 2008, 31, 1275-1289. [CrossRef]

27. Zhou, S.; Hu, W.; Deng, X.; Ma, Z.; Chen, L.; Huang, C.; Wang, C.; Wang, J.; He, Y.; Yang, G.; et al. Overexpression of the wheat aquaporin gene, $\mathrm{Ta} A Q P 7$, enhances drought tolerance in transgenic tobacco. PLoS ONE 2012, 7, e52439. [CrossRef] 
28. Hu, W.; Yuan, Q.; Wang, Y.; Cai, R.; Deng, X.; Wang, J.; Zhou, S.; Chen, M.; Chen, L.; Huang, C.; et al. Overexpression of a wheat aquaporin gene, TaAQP8, enhances salt stress tolerance in transgenic tobacco. Plant Cell Physiol. 2012, 53, 2127-2141. [CrossRef]

29. Hanba, Y.T.; Shibasaka, M.; Hayashi, Y.; Hayakawa, T.; Kasamo, K.; Terashima, I.; Katsuhara, M. Overexpression of the barley aquaporin $\mathrm{HvPIP2;1}$ increases internal $\mathrm{CO}_{2}$ conductance and $\mathrm{CO}_{2}$ assimilation in the leaves of transgenic rice plants. Plant Cell Physiol. 2004, 45, 521-529. [CrossRef]

30. Geng, H.W.; Shi, L.; Li, W.; Zhang, B.; Chu, C.C.; Li, H.J.; Zhang, G.F. Gene expression of jojoba (Simmondsia chinensis) leaves exposed to drying. Environ. Exp. Bot. 2008, 63, 137-146. [CrossRef]

31. Ayadi, M.; Cavez, D.; Miled, N.; Chaumont, F.; Masmoudi, K. Identification and characterization of two plasma membrane aquaporins in durum wheat (Triticum turgidum L. subsp. durum) and their role in abiotic stress tolerance. Plant Physiol. Biochem. 2011, 49, 1029-1039.

32. Polle, A. Dissection the superoxide dismutase-ascorbate-glutathione pathway by metabolic modeling: Computer analysis as a step towards flux analysis. Plant Physiol. 2001, 126, 445-462. [CrossRef]

33. Mittler, R.; Vanderauwera, S.; Gollery, M.; Van Breusegem, F. Reactive oxygen gene network of plants. Trends Plant Sci. 2004, 9, 490-498. [CrossRef]

34. Moore, K.; Roberts, L.J. Measurement of lipid peroxidation. Free Radic. Res. 1998, 28, 659-671. [CrossRef]

35. Xu, Y.; Hu, W.; Liu, J.; Zhang, J.; Jia, C.; Miao, H.; Xu, B.; Jin, Z. A banana aquaporin gene, MaPIP1;1, is involved in tolerance to drought and salt stresses. BMC Plant Biol. 2014, 14, 59. [CrossRef]

36. Li, G.W.; Zhang, M.H.; Cai, W.M.; Sun, W.N.; Su, W.A. Characterization of OsPIP2;7, a water channel protein in rice. Plant Cell Physiol. 2008, 49, 1851-1858. [CrossRef]

37. Liu, J.; Zhu, J.K. Proline accumulation and salt-stress-induced gene expression in a salt-hypersensitive mutant of Arabidopsis. Plant Physiol. 1997, 114, 591-596. [CrossRef]

38. Livak, K.J.; Schmittgen, T.D. Analysis of relative gene expression data using real-time quantitative PCR and the $2^{-\Delta \Delta C t}$ method. Methods 2001, 25, 402-408. [CrossRef]

39. Clough, S.J.; Bent, A.F. Floral dip: A simplified method for Agrobacterium mediated transformation of Arabidopsis thaliana. Plant J. 1998, 16, 735-743. [CrossRef]

40. Zhang, L.; Xiao, S.; Li, W.; Feng, W.; Li, J.; Wu, Z.; Gao, X.; Liu, F.; Shao, M. Overexpression of a Harpin-encoding gene $h r f 1$ in rice enhances drought tolerance. J. Exp. Bot. 2011, 62, 4229-4238. [CrossRef]

41. Jiang, M.; Zhang, J. Effect of abscisic acid on active oxygen species, antioxidative defence system and oxidative damage in leaves of maize seedlings. Plant Cell Physiol. 2001, 42, 1265-1273. [CrossRef] [PubMed] 